\title{
Anomalous nucleation far from equilibrium
}

\author{
I. T. Georgiev, B. Schmittmann, and R. K. P. Zia \\ Center for Stochastic Processes in Science and Engineering and \\ Department of Physics, Virginia Tech, Blacksburg, VA 24061-0435 USA
}

(Dated: November 26, 2018)

\begin{abstract}
We present precision Monte Carlo data and analytic arguments for an asymmetric exclusion process, involving two species of particles driven in opposite directions on a $2 \times L$ lattice. We propose a scenario which resolves a stark discrepancy between earlier simulation data, suggesting the existence of an ordered phase, and an analytic conjecture according to which the system should revert to a disordered state in the thermodynamic limit. By analyzing the finite size effects in detail, we argue that the presence of a single, seemingly macroscopic, cluster is an intermediate stage of a complex nucleation process: In smaller systems, this cluster is destabilized while larger systems allow the formation of multiple clusters. Both limits lead to exponential cluster size distributions which are, however, controlled by very different length scales.
\end{abstract}

PACS numbers: 64.60.Cn, 64.75.+g, 68.43.Jk

Introduction. From recent studies of statistical model systems far from thermal equilibrium, it is clear that even their stationary states pose many new challenges. The well established theoretical machinery for systems in thermal equilibrium is essentially powerless in these contexts, and our equilibrium-trained intuition often misleads us. For example, though the specific heat of an equilibrium system cannot be negative, the internal energy of a non-equilibrium steady state may decrease when coupled to a thermal bath at a higher temperature [1. In general, non-equilibrium steady states depend sensitively on the details of the microscopic dynamics, resulting in widely diverse behavior at the macroscopic level. So far, an overarching framework is still lacking, but much insight has been gained by studying simple model systems.

One such class of models are driven diffusive systems [2]. Close relatives of the asymmetric exclusion process [3], they involve interacting diffusing particles, driven into selected spatial directions by an external force. Models of this kind have been invoked to describe vehicular and pedestrian traffic [4, gel electrophoresis 5], and a wide range of biological problems, ranging from molecular motors [6] to protein synthesis 7]. A particularly rich phase diagram emerges for two species of particles, driven in opposite directions on a two-dimensional $(2 D)$ periodic lattice [8]. Even with no interaction except excluded volume, we observe transitions from a freely flowing homogeneous state to a "jammed" state displaying a macroscopic cluster of particles. These transitions persist even if neighboring particles are allowed to exchange places, with a small rate $\gamma[9]$. In contrast, an analytic solution [10] for the same system in $1 D$ shows that the homogeneous state prevails always, for any $\gamma>0$ and particle density $\rho<1$. Exploring notions of a lower critical dimension, we studied a quasi- $1 D$ system, involving just two "lanes", i.e., $2 \times L$, with $L$ up to $10^{4}$ 11, 12. Remarkably, for small $\gamma$ and half-filling, the jammed state re-emerged, with the length of the jam scaling with $L$. However, it was argued recently [13] that this jam is a mere finite-size effect and should break up into a disordered state when $L$ exceeds a characteristic crossover length $L_{c}$. While the latter is not known exactly, it might be as large as $10^{70}$ [14!

Since simulations are an essential tool in the study of nonequilibrium steady states and should provide a fairly accurate picture of the thermodynamic limit, such stark discrepancies between simulation data and analytic arguments are disconcerting and need to be explored further. In this letter, we report extensive, high precision computer simulations, studying the full $(\rho, \gamma)$ space and a wide range of $L$ for this two-lane system. Defining an "s-cluster" as $s$ particles of either species, connected to each other via nearest-neighbor bonds, we focus on the normalized residence distribution, $p(s)$, which is the probability that a randomly chosen particle belongs to an $s$-cluster. For a homogeneous state, $p(s)$ is monotonically decreasing. In contrast, for a jammed system it displays two distinct peaks: one at the origin $(s=1)$ and another marking the macroscopic cluster, at $s_{o} \propto L$ [1]. If a crossover length $L_{c}$ exists, this second peak should disappear when $L$ increases beyond $L_{c}$. Expecting $L_{c}$ to decrease with $\gamma$, we consider much larger $\gamma$ 's than in previous studies [1] and find, indeed, that systems with $\rho>0.3$ cross over to monotonic distributions with increasing $L$. However, at lower densities (e.g., $\rho=0.1$ ) we discover the opposite behavior: here, $p(s)$ is monotonic at small $L$ and develops a second peak as $L$ becomes larger. In order to interpret these complex finite-size effects, we propose a new picture, in which larger clusters compete with a homogeneous background of "travellers", consisting only of isolated particles and very small clusters. In the remainder of this letter, we briefly review the model, present the simulation results, and provide the analysis that leads us to our conclusions.

The model. In a $2 \times L$ lattice with fully periodic boundary conditions, each site can be either empty $(\oslash)$ or occupied by a single particle, which we identify as either "positive" $(+)$ or "negative" $(-)$. Configurations are labeled by $\left\{\sigma_{x, y}\right\}$, where $\sigma$ takes on values $0, \pm 1$ and $x \in[1, L] ; y=1,2$. The two species are "driven" in opposite directions, much 

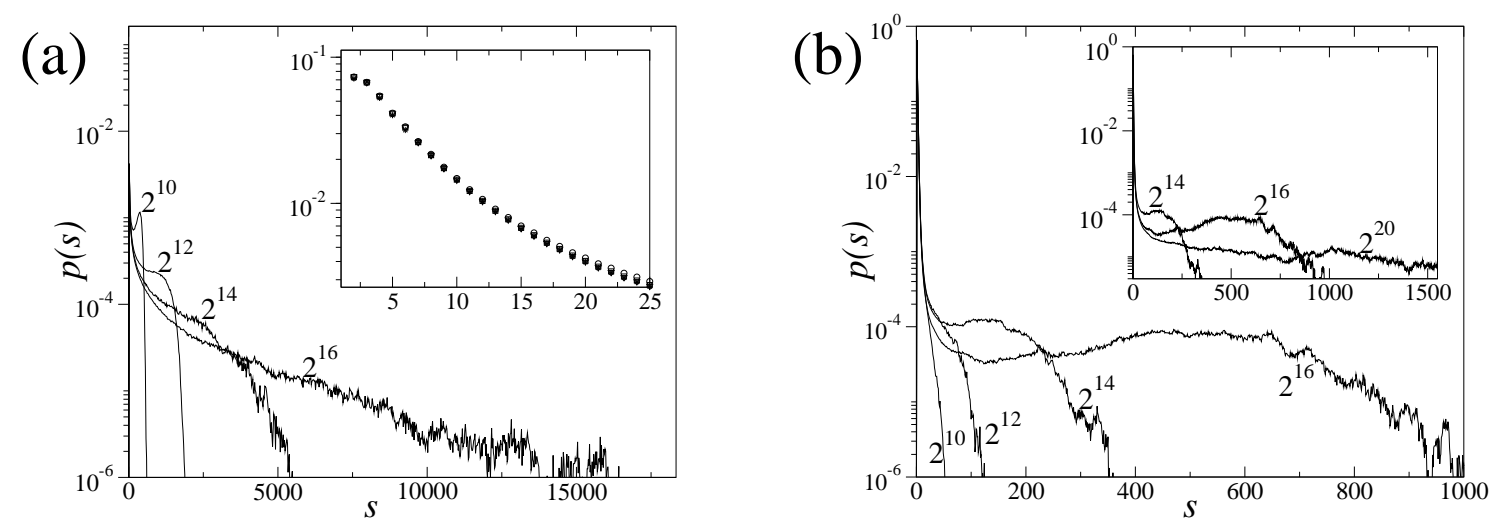

FIG. 1: The residence distribution $p(s)$ vs. $s$ on lattices with $L=2^{10}, 2^{12}, 2^{14}$ and $2^{16}$ for (a) $\rho=0.5$ and $\gamma=0.45$ and (b) $\rho=0.1$ and $\gamma=0.163$. The inset on the left figure magnifies the small-s data and the inset on the right shows the change from a double-peaked to a single-peaked distribution by increasing $L$.

like cars on a 2-lane road. Starting from an initial configuration with equal numbers of the two species occupying a fraction, $\rho$, of the sites (i.e., $N_{+}=N_{-}=\rho L$ ), we evolve the system by exceedingly simple rules. In one Monte Carlo step (MCS), the following steps are repeated $2 L$ times: $(i)$ a pair of two nearest-neighbor sites (a "bond") is chosen at random; ( $i$ i) for bonds along the $L$ direction, a $(+\oslash)$ or a $(\oslash-)$ pair is always exchanged while a $(+-)$ pair is exchanged with probability $\gamma$; (iii) for transverse bonds, particle-hole pairs are always exchanged while particle-particle pairs are exchanged with probability $\gamma$. Other moves are not allowed.

To probe the system's behavior and the effects of finite size, we explore a wide range of $\gamma$ and $\rho$, and lattices with $L=2^{8}, 2^{10}, 2^{12}, 2^{14}$, and $2^{16} \simeq 64 K$. Since our main focus is the steady state, we discard up to $2 \times 10^{6}$ MCS before taking measurements. Thereafter, quantities of interest are recorded every $100 \mathrm{MCS}$, for up to $10^{7} \mathrm{MCS}$. On a 64-bit machine, we exploit a fast multispin coding algorithm (e.g., 15]) by encoding the state on a site for 64 different lattices (realizations) simultaneously. Thus, we are confident that our statistical errors are minimal.

Simulation results. We set the scene by presenting a characteristic data set for the residence distribution $p(s)$ at $\rho=0.5$ and $\gamma=0.45$ (Fig. [1 $)$, in order to demonstrate the crossover from bimodal to single-peaked distributions with increasing $L$. The smallest system, $L=2^{10}$, shows a distinct peak at $s_{o} \simeq 380$, while the next size, $L=2^{12}$, shows only a shoulder which broadens into a well-defined exponential, $\propto \exp [-s \xi(\gamma, \rho)]$, with a 'slope' $\xi(0.45,0.5) \simeq 0.25 \times 10^{-3}$ for the largest system. It is remarkable that, even for such large $L$ 's, the large $s$ data still depend quite sensitively on $L$. On the other hand, as the inset shows, the small $s$ part is manifestly independent of $L$, a feature that persists in the whole $(\gamma, \rho)$ space. Since we expect the crossover length $L_{c}$ to decrease with $\gamma$, we consider systems with $\gamma=0.5$, keeping $\rho$ fixed at 0.5 . Here, even the smallest system $\left(L=2^{10}\right)$ already displays a monotonic distribution. In contrast, if $\gamma=0.4$, the distributions remain double-peaked for all $L \leq 2^{16}$. In fact, for those $L$, the (second) peak position scales as $s_{o} \sim 0.8 L^{0.95}$ which one might (naively) interpret as the signature of a "macroscopic" cluster, unless even larger $L$ are investigated. To summarize, these findings are consistent with the conjecture [13] that the presence of 'macroscopic' clusters is a finite-size effect. For $L \rightarrow \infty$, we may expect residence distributions to comprise two components: one at small $s$ (which we associate with the "travellers") and a slow exponential decay $\exp [-s \xi(\gamma, \rho)]$ (associated with large scale "jams").

Surprisingly, the behavior of our model is richer than this simple picture suggests. For smaller density and $\gamma$, there is an additional crossover, in the opposite direction: Distributions are monotonic in small $L$ systems and become bimodal as $L$ increases, only reverting to being monotonic for much larger $L$. The first of these crossovers is illustrated in Fig. 1b, which shows residence distributions for the same four $L$ 's but at $\rho=0.1$ and $\gamma=0.163$. We re-emphasize: In contrast to Fig. 1 $\mathrm{a}$, here the second peak emerges with increasing $L$.

Fig. 1 suggests that, for any given $L$, it is possible to identify two distinct regions in $(\gamma, \rho)$ space: In one of them, located near the large $\gamma$, small $\rho$ corner, $p(s)$ is monotonically decreasing in $s$; in the other region (small $\gamma$, larger $\rho$ ), $p(s)$ is bimodal. To summarize our data, the corresponding boundaries, $\gamma_{c}(\rho, L)$, are drawn in Fig. 2 for several $L$. If larger systems favor (disfavor) bimodal distributions, $\gamma_{c}(\rho, L)$ shifts up (down) with $L$.

Analytic arguments. In the following, we present a simple scenario which encompasses the full range of these phenomena. Our discussion begins at very low densities. A space-time plot (Fig. 3 3 ) for $\rho=0.1, \gamma=0.1$, and $L=2^{8}$ shows the evolution of a typical configuration. With most of the system occupied by travellers, local density fluctuations occasionally "nucleate" a single larger cluster, containing about $24 \pm 8$ particles, which survives for up to $1000 \mathrm{MCS}$, then disintegrates and reforms elsewhere. When one of these large clusters is present, the background 


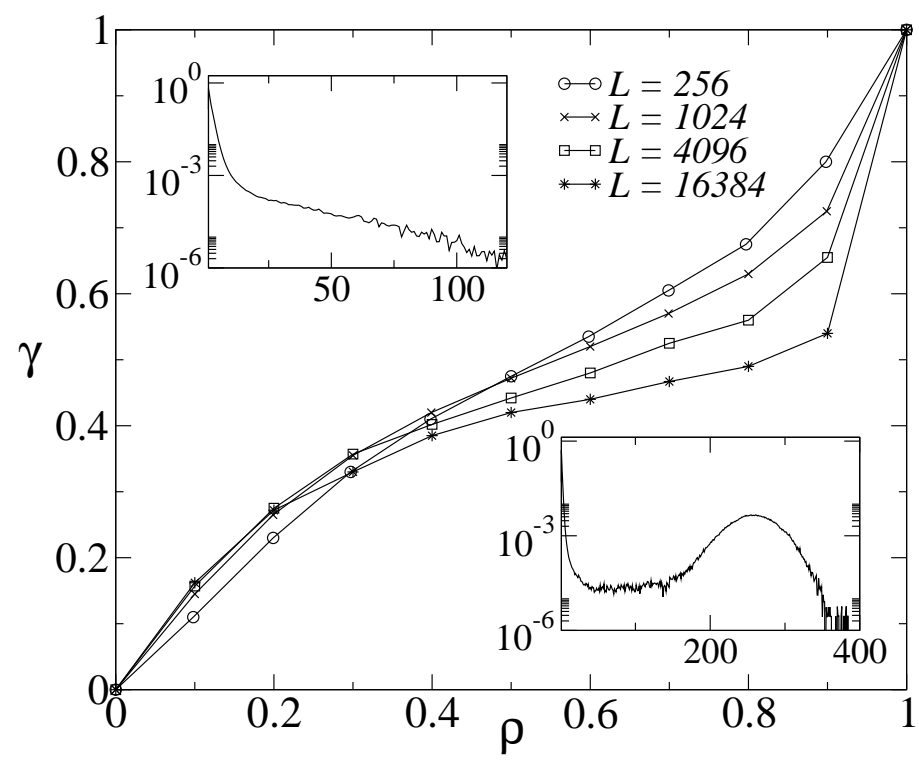

FIG. 2: Boundaries separating monotonic from bimodal distributions, for various $L$. The insets show $p(s)$ for $\rho=0.1, \gamma=0.3$ (top left) and $\rho=0.1, \gamma=0.125$ (bottom right) on a $L=2^{12}$ lattice.

density of travellers is depleted noticeably. Reducing $\gamma$ lengthens the cluster life times, until a single cluster persists for the whole run. In contrast, at higher $\gamma$, large clusters just blink on and off, and the traveller component dominates.

Motivated by these observations, we describe the system at such low densities in terms of two "components". One of these is purely uniform, consisting only of travellers at density $\rho$. The other exhibits a single large cluster, of mass $s$, reducing the density of travellers in the remainder of the system to $\rho(s)=(2 L \rho-s) /(2 L-s)$. In the spirit of mean-field theory, we neglect fluctuations in the cluster size as well as any charge asymmetries. Since the total number of each species is conserved, it is natural to focus on, e.g., the current of positive particles through either lane, as a function of the local densities. Of course, the current in the interior of a cluster, $j_{c}(s)$, should display a very different density dependence from the current carried by the travellers, $j_{t}(s)$. Assuming the traveller region to be approximately uniform, $j_{t}(s)$ should be well represented by its mean-field form $[9], j_{t}(s)=\rho(s)[1-\rho(s)] / 2+(\gamma / 4) \rho^{2}(s)$. Here, the first term reflects particle-hole exchanges, proportional to the density of positive particles, $\rho(s) / 2$, and that of holes, $1-\rho(s)$. The second term models the charge exchanges. For the current through a cluster of size $s$, the exact form is known only for the one-lane model, $j_{c}(s)=(\gamma / 4)\left[1+b / s+O\left(s^{-2}\right)\right]$ with $b=3 / 2[13]$. For the two-lane system, we have to rely on simulations which confirm the same form up to a slightly modified coefficient, $b \simeq 1.6[13$, 16]. Even though the $O\left(s^{-2}\right)$ corrections are not taken into account, this form appears to be sufficiently accurate for our purposes.

Clearly, a large cluster of size $s$ can co-exist with a uniform region only if the two currents, $j_{c}(s)$ and $j_{t}(s)$, are equal. If $j_{c}(s)>j_{t}(s)$, the cluster will lose particles and shrink; for $j_{c}(s)<j_{t}(s)$, it will grow in size. This allows us to identify both stable and unstable solutions of $j_{c}(s)=j_{t}(s)$, as functions of $\gamma, \rho$, and $L$, in the physical domain $s \geq 1$. Leaving details to a future publication [17], we just outline the main qualitative predictions here. To begin with, we note that $j_{c}(s)$ is independent of $\rho$ and $L$ while $j_{t}(s)$ varies only very weakly with $\gamma$. In other words, we can plot $j_{t}(s)$ for a given $(\rho, L)$ and then watch $j_{c}(s)$ shift up or down, proportional to $\gamma$. In this fashion, we can easily identify three regimes: $(i)$ At small $\rho$ and $L$, and $\gamma$ sufficiently large, no solution exists: $j_{t}(s)$ remains well below $j_{c}(s)$, and the system is homogeneously filled with travellers. While local fluctuations occasionally generate small clusters, these disintegrate almost immediately. The two smallest system sizes of Fig. 1p belong into this regime. (ii) As $\gamma$ decreases, the two curves $j_{t}(s)$ and $j_{c}(s)$ first touch, and then two solutions emerge: an unstable one $\left(s_{u}\right)$ close to the origin which plays the role of a critical droplet, and a stable one at $s_{o}>s_{u}$ which sets the cluster size. If $s_{o}$ is not much larger than $s_{u}$ and fluctuations are included, we expect to see purely uniform configurations compete with those supporting one larger cluster, as in Fig. 3 k. For those parameter values, we predict $s_{o}=24$, in remarkably good agreement with the data. Returning to Fig. 1b, we note that two solutions can also emerge if $L$ increases at fixed $\gamma$, as illustrated by the $L=2^{12}$ data. Analytically, we expect $s_{o}=70$ here. Since the two currents differ by less than 5 up to $s \simeq 120$, clusters of all these sizes form quite easily, giving rise to the observed $p(s)$. (iii) Finally, as $\gamma$ is lowered further, only the stable solution survives and shifts to $s_{o} \sim O(L)$. Before this occurs, however, fluctuations begin to play a much more important role: Considering the $L=2^{16}$ system in Fig. 1b, our theory would predict $s_{o}=1436$, 

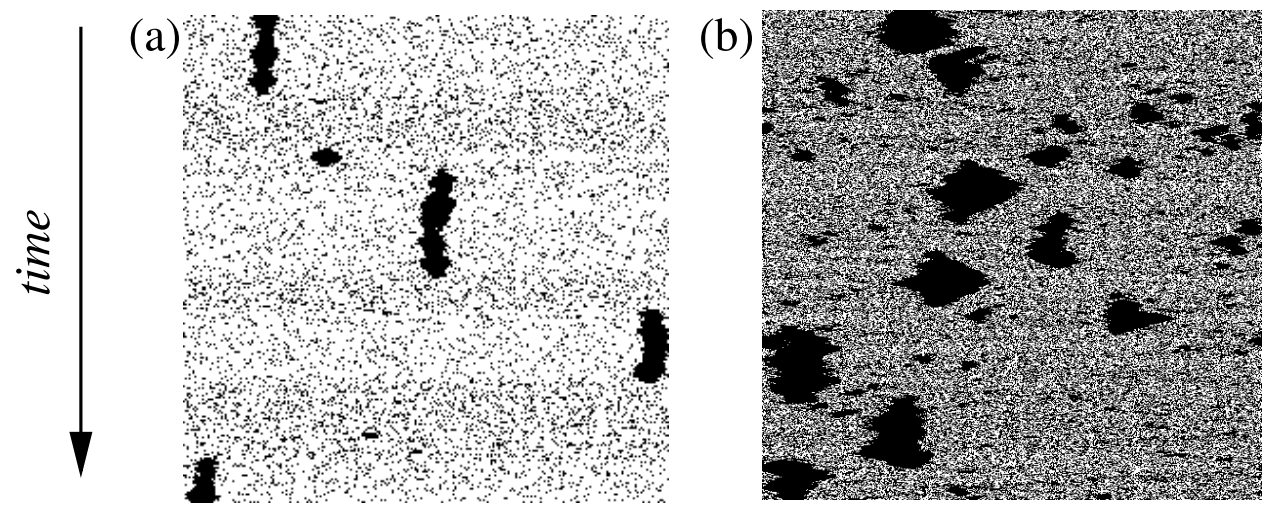

FIG. 3: Space-time plot at $\rho=0.1, \gamma=0.1, L=2^{8}$ (a) and $\rho=0.4, \gamma=0.4$, and $L=2^{9}$ (b). The spatial (temporal) coordinate runs horizontally (vertically, in units of $50 \mathrm{MCS}$ ). A black dot indicates a particle in either lane.

but the largest observed clusters remain well below 700 particles. Monitoring typical configurations, we discover that the system is attempting to nucleate a second cluster in the traveller region, in addition to a persistent first one.

To summarize, our simple mean-field theory describes the "nucleation" of a single cluster, from a uniform background of travellers, remarkably well. Naturally, once a single cluster exists, and either $\rho$ or $L$ are increased (or $\gamma$ reduced), we should expect, and do indeed observe, the "nucleation" of additional clusters in the traveller region. As this process continues, the residence distribution broadens, and eventually crosses over to a slow exponential decay, as in Fig. 19. To illustrate this, we present a space-time plot for $\rho=0.4, \gamma=0.4$ and $L=2^{9}$ (Fig. 3b) which shows several clusters of different sizes in each time slice.

Conclusions. We have presented extensive simulation data for a $2 \times L$ system with two species of particles driven in opposite directions. Varying $L$, density $\rho$, and the particle exchange rate $\gamma$, we monitor the cluster size ("residence") distribution $p(s)$. We find two well-separated domains in $(\rho, \gamma)$ space: one at low $\rho$, high $\gamma$ with monotonically decreasing $p(s)$, and one at high $\rho$, low $\gamma$ with bimodal distributions. The boundary between these domains shifts with $L$, the general trend favoring monotonic $p(s)$ as $L$ increases.

To understand the underlying physics at the crossovers, we draw some insight from nucleation processes. For any fixed value of $\gamma$, systems are uniform for sufficiently low $\rho$ and $L$, and the associated residence distribution falls off rapidly. As either $\rho$ or $L$ increases, a single large cluster is nucleated. The residence distribution develops a distinct shoulder and, eventually, a second peak. This transition is well captured by a simple mean-field theory which balances the current through the cluster with that through the uniform region. As $\rho$ or $L$ increase further, a second cluster is nucleated, and eventually a whole population is established. As a result, the residence distribution becomes monotonic again, exhibiting a slow exponential decay.

With presently accessible $L$ 's, we may only conclude that our data for $\gamma_{c}(\rho, L)$ (cf. Fig. 3) is consistent with the conjecture [13]. At the same time, given the absence of an exact solution, we cannot rule out the possibility that $\gamma_{c}(\rho, L)$ might have a non-trivial $L \rightarrow \infty$ limit. We do stress, however, that its non-monotonic $L$-dependence (at fixed $\rho)$ appears unprecedented in equilibrium nucleation [18] or phase separation [19] processes. Whatever the eventual conclusion, we believe that it is important to explore finite-size effects, since these are relevant for most applications of interest. For example, in the modelling of protein synthesis, system sizes ranging from $1 \mathrm{~K}$ to $10 \mathrm{M}$ cover the typical lengths of mRNA molecules. In these contexts, we feel that both crossovers may be of theoretical as well as practical interest.

Acknowledgements. We thank J.T. Mettetal and M. Mobilia for stimulating discussions. This research is supported in part by the US National Science Foundation through grants DMR-0088451 and DMR-0414122.

[1] R.K.P. Zia, E.L. Praestgaard, and O.G. Mouritsen, Am. J. Phys. 70, 384 (2002).

[2] S. Katz, J.L. Lebowitz, and H. Spohn, Phys. Rev. B 28, 1655 (1983) and J. Stat. Phys. 34, 497 (1984). For reviews, see, e.g., B. Schmittmann and R.K.P. Zia, Statistical mechanics of driven diffusive systems. In: Phase Transitions and Critical Phenomena, Vol. 17, ed. by C. Domb and J.L. Lebowitz (Academic Press, New York 1995); D. Mukamel, Phase transitions in non-equilibrium systems, In: Soft and Fragile Matter: Nonequilibrium Dynamics, Metastability and Flow, eds. M.E. Cates and M.R. Evans (IOP Publishing, Bristol, 2000).

[3] J.T. MacDonald, J.H. Gibbs, and A.C. Pipkin, Biopolymers 6, 1 (1968); for a review, see G.M. Schütz, Integrable stochastic many-body systems. In: Phase Transitions and Critical Phenomena, Vol. 19, ed. by C. Domb and J.L. Lebowitz (Academic 
Press, New York 2001).

[4] O. Biham, A.A. Middleton, and D. Levine: Phys. Rev. A 46, R6124 (1992); D. Chowdhury, L. Santen, and A. Schadschneider: Phys. Rep. 199 (2000).

[5] M. Rubinstein: Phys. Rev. Lett. 59, 1946 (1987); T.A.J. Duke: Phys. Rev. Lett. 62, 2877 (1989); B. Widom, J.L. Viovy, and A.D. Desfontaines: J. Phys I (France) 1, 1759 (1991); U. Alon and D. Mukamel Phys. Rev. E 55, 1783 (1997).

[6] J. Howard, Nature 389, 561 (1997); M.E. Fisher and A.B. Kolomeisky, Proc. Natl. Acad. Sci. USA 96, 6597 (1999).

[7] L.B. Shaw, R.K.P. Zia, and K.H. Lee, Phys. Rev. E 68, 021910 (2003); MacDonald et.al. in [3].

[8] B. Schmittmann, K. Hwang and R.K.P. Zia, Europhys. Lett. 19, 19 (1992).

[9] G. Korniss, B. Schmittmann and R.K.P. Zia, J. Stat. Phys. 86, 721 (1997).

[10] S. Sandow and C. Godrèche, unpublished (1998).

[11] G. Korniss, B. Schmittmann and R.K.P. Zia, Europhys. Lett. 45, 431 (1999).

[12] J. T. Mettetal, B. Schmittmann and R.K.P. Zia, Europhys. Lett. 58, 653 (2002); B. Schmittmann, J.T. Mettetal and R.K.P. Zia, in Computer Simulation Studies in Condensed Matter Physics XVI, eds. D.P. Landau, S.P. Lewis, and H.B. Schüttler (Springer, in press).

[13] Y. Kafri, E. Levine, D. Mukamel, G.M. Schütz and J. Török, Phys. Rev. Lett. 89, 035702 (2002); Y. Kafri, E. Levine, D. Mukamel and J. Török, J. Phys. A: Math. Gen. 35, L459 (2002).

[14] N. Rajewsky, T. Sasamoto and E.R. Speer, Physica A 279, 123 (2000); T. Sasamoto and D. Zagier, J. Phys. A: Math. Gen. 34, 5033 (2001).

[15] M.E.J. Newman and G.T. Barkema, Monte Carlo Methods in Statistical Physics (OUP, 1999).

[16] J.T. Mettetal, B. Schmittmann and R.K.P. Zia, unpublished; M.R. Evans, E. Levine, P.K. Mohanty, and D. Mukamel, cond-mat/0405049

[17] I. Georgiev, B. Schmittmann and R.K.P. Zia, in preparation.

[18] P.A. Rikvold, H. Tomita, S. Miyashita and S.W. Sides, Phys. Rev. E 49, 5080 (1994).

[19] A. Christensen, P. Stoltze and J.K. Norskov, J. Phys.: Cond. Mat. 7, 1047 (1995). 